\title{
SELEÇÃO DE ESPÉCIES BIOINDICADORAS PARA O HERBICIDA INDAZIFLAM
}

\section{SELECTION OF BIOINDICATOR SPECIES FOR THE HERBICIDE INDAZIFLAM}

Roque Carvalho Dias ${ }^{\mathrm{a} *}$, Diego Munhoz Gomes ${ }^{\mathrm{a}}$, Vitor Muller Anunciato ${ }^{\mathrm{a}}$, Leandro Bianchi ${ }^{\mathrm{a}}$, Plinio Saulo Simões ${ }^{\mathrm{b}}$, Caio Antonio Carbonari ${ }^{\mathrm{b}}$, Edivaldo Domingues Velini ${ }^{\mathrm{b}}$

aDepartamento de Proteção de Plantas, Universidade Estadual Paulista, Faculdade de Ciências Agronômicas, São Paulo, Brasil. bDepartamento de Produção e Melhoramento Vegetal, Universidade Estadual Paulista, Faculdade de Ciências Agronômicas, São Paulo, Brasil.

*Autor correspondente: roquediasagro@gmail.com.

\section{INFORMAÇÕES DO ARTIGO Histórico do artigo:}

Recebido: 24 Novembro 2018.

Aceito: 13 Junho 2019.

Publicado: 25 Agosto 2019

Palavras-chave/Keywords:

Inibidor da Síntese de Parede Celular/ Cellulose Biosynthesis Inhibitor. Alquilazina/ Alkylazine.

\section{Financiamento:}

O presente trabalho foi realizado com apoio da Coordenação de Aperfeiçoamento de Pessoal de Nível Superior - Brasil (CAPES) - Código de Financiamento 001.

Ao Conselho Nacional de Pesquisa (CNPq) pelo apoio financeiro a pesquisa através do Projeto (Processo n. 140683/2018-6).

Direito Autoral: Este é um artigo de acesso aberto distribuído sob os termos da Licença Creative Commons, que permite uso, distribuição e reprodução irrestritos em qualquer meio, desde que $\mathrm{o}$ autor e a fonte originais sejam creditados.

\section{Citação deste artigo:}

DIAS, R. C.; GOMES, D. M.; ANUNCIATO, V. M.; BIANCHI, L.; SIMÕES, P. S.; CARBONARI, C. A.; VELINI, E. D. Seleção de espécies bioindicadoras para o herbicida indaziflam. Revista Brasileira de Herbicidas, v. 18, n.2. 2019.

\begin{abstract}
RESUMO
O herbicida indaziflam apresenta atividade residual no solo e pode se tornar problema para os cultivos agrícolas, pela possibilidade de carryover em culturas sucessoras. Assim, objetivou-se selecionar potenciais espécies bioindicadoras para presença de resíduos do indaziflam no solo. Foram realizados dois experimentos em casa de vegetação, utilizando vasos de polietileno de $0,00025 \mathrm{~m}^{3}$, ambos distribuídos no delineamento em blocos ao acaso com quatro repetições. No primeiro experimento foram utilizadas onze espécies com potencial uso como bioindicadoras: arroz, aveia, beterraba, capim-braquiária, feijão, girassol, milho, pepino, soja, tomate e trigo submetidas à aplicação de sete doses de indaziflam $\left(0 ; 6,25 ; 12,5 ; 25 ; 50 ; 75\right.$ e $100 \mathrm{~g}$ i.a. ha $\left.{ }^{-1}\right)$. em pré-emergência das culturas. No segundo experimento, cinco sub-doses $\left(0 ; 0,3906 ; 0,7812 ; 1,5625\right.$ e 3,125 g i.a. ha $\left.^{-1}\right)$ do indaziflam foram aplicadas nas espécies que não emergiram na menor dose ou concentração do herbicida no primeiro experimento. Foram avaliadas a fitointoxicação e altura de plantas (ALT) aos 3, 7, 14 e 21 dias após a emergência (DAE). Aos 21 DAE avaliou-se matéria seca de parte aérea (MSPA), de raiz (MSR) e total (MST). O aumento das doses de indaziflam provocou aumento dos sintomas de fitointoxicação e reduções nas variáveis ALT, MSPA, MSR e MST. Entre as espécies avaliadas, em monocotiledôneas na ordem: capim-braquiária, arroz, milho, trigo, aveia e em eudicotiledôneas: tomate, pepino, girassol, feijão, soja apresentam potencial para uso como bioindicadora em estudos com indaziflam. A beterraba apresentou ser extremamente sensível ao herbicida não emergindo em nenhuma das doses estudadas.
\end{abstract}




\section{Introdução}

Os herbicidas, depois de aplicados, podem atingir o solo ou ser absorvidos pela parte aérea da planta. Quando entram em contato com o solo, eles estão sujeitos à sorção, transformação e processos de transporte, e estes compostos podem persistir por um curto período, por meses ou anos, no caso daqueles altamente persistente (AMIM et al., 2014). Esse comportamento pode variar de acordo as características físico-químicas do herbicida e do solo, umidade do solo, atividade microbiana e condições climáticas (INOUE et al., 2011).

A atividade residual de herbicidas no solo quando bem manejada e ativa na solução do solo, possibilita suprimir novos fluxos de plantas daninhas, reduzindo o número de aplicações e permitindo que a cultura possa emergir em ambiente com competição mínima ou nula. No entanto, os resíduos de herbicidas no solo podem levar à intoxicação e prejudicar o crescimento e desenvolvimento de culturas em sucessão (carryover) (MELO et al., 2016).

O herbicida indaziflam pertence ao grupo químico da alquilazina, e atua como um inibidor de biossíntese de celulose, é recomendado para a aplicação em préemergência no controle de monocotiledôneas e eudicotiledôneas em diversas culturas como: banana, café, caju, cana-de-açúcar, citro, coco, entre outras (MAPA, 2018). As características fisico-químicas são a baixa solubilidade em água $\left(0,0028 \mathrm{~kg} \mathrm{~m}^{-3}\right.$ a $\left.20^{\circ} \mathrm{C}\right)$, o Koc $<1.000$ $\mathrm{mL} \mathrm{g}^{-1}$ de carbono orgânico, o Pka de 3,5 e o log Kow em pH 4,7 ou 9 de 2,8 (TOMPKINS, 2010). O indaziflam é caracterizado pela meia-vida de 150 dias, alta persistência no solo (ALONSO et al., 2015, (KAAPRO; HALL, 2012), em soja e beterraba a persistência da atividade biológica do indaziflam foi superior a 150 dias tanto em solo muito argiloso quanto em textura franco-argiloarenosa (GUERRA et al., 2016). Segundo Guerra et al. (2013) os metabólitos desse herbicida apresentam alto potencial de lixiviação para água subterrânea, o que faz necessário conhecimento do seu comportamento no solo.

Dentre os métodos de monitoramento e avaliação de resíduos de herbicidas no solo, destaca-se a técnica de bioensaio, na qual utilizam-se plantas que demonstram alta sensibilidade ao produto avaliado e apresenta baixo custo em relação as demais técnicas, fácil cultivo e rápido desenvolvimento (DIAS et al., 2017). Dessa forma, diante da simplicidade e baixo custo para realização torna-se uma das técnicas mais comuns na detecção de resíduos de herbicidas no solo (NUNES; VIDAL, 2009).

Considerando o tempo de permanência desse herbicida no solo e a possibilidade de contaminação de cultura sucessoras, objetivou-se neste trabalho selecionar espécies com potencial uso como bioindicadoras para indicação de resíduos de indaziflam no solo.

\section{Material e Métodos}

Foram realizados dois experimentos em casa de vegetação, em delineamento em blocos ao acaso, com quatro repetições, utilizando-se como substrato Latossolo Vermelho Distroférrico (EMBRAPA, 2013), de textura média cuja as propriedades físico-químicas encontram-se na Tabela 1, as amostras de solo foram coletadas na Fazenda Experimental Lageado em Botucatu-SP em uma área em pousio, na profundidade de $0-20 \mathrm{~cm}$. As amostras de solo foram peneiradas para remoção dos resíduos presentes na superfície do solo, e posteriormente secas ao ar e acondicionadas em vasos de polietileno com capacidade de $0,00025 \mathrm{~m}^{3}$.

Tabela 1. Características físico-químicos do Latossolo Vermelho Distroférrico de textura média utilizado no experimento

\begin{tabular}{ccccccc}
\hline \multicolumn{1}{c}{$\begin{array}{c}\mathrm{pH} \\
\left(\mathrm{CaCl}_{2}\right)\end{array}$} & & $\mathrm{Al}^{3+}$ & $\begin{array}{c}\mathrm{H}^{+}+\mathrm{Al}^{3+} \\
\left(\mathrm{mmol}_{\mathrm{c}} \mathrm{dm}^{-3}\right)\end{array}$ & $\mathrm{Ca}^{2+}+\mathrm{Mg}^{2+}$ & $\mathrm{Ca}^{2+}$ & $\mathrm{K}^{+}$ \\
\cline { 3 - 5 } & & 0,00 & 41,00 & 4,00 & 3,00 & 0,10 \\
\hline M.O. & $\mathrm{P}$ & $\mathrm{CTC}(\mathrm{pH}=7)$ & $\mathrm{V}$ & Areia & Silte & Argila \\
$\left(\mathrm{g} \mathrm{dm}^{-3}\right)$ & $\left(\mathrm{mg} \mathrm{dm}^{-3}\right)$ & $\left(\mathrm{mmol}_{\mathrm{c}} \mathrm{dm}^{-3}\right)$ & $(\%)$ & & $(\mathrm{g} \mathrm{kg})$ & \\
17,00 & 1,00 & 55,00 & 58,00 & 644 & 47 & 309 \\
\hline
\end{tabular}

No primeiro experimento os tratamentos foram constituídos de sete doses do herbicida indaziflam (Alion ${ }^{\circledR}$, $\mathrm{SC}, 500$ g i.a. L, Bayer S.A.) $0 ; 6,25 ; 12,5 ; 25 ; 50 ; 75$ e 100 g i.a. ha ${ }^{-1}$, o que equivale a $0 ; 6,25 ; 12,5 ; 25 ; 50 ; 75 ; 100 \%$ da dose recomendada pelo fabricante. Foi realizado um segundo experimento para as espécies que não emergiram na menor dose e/ou concentração do herbicida, para isso foram utilizadas cinco doses do indaziflam: $(0 ; 0,3906$; 0,$7812 ; 1,5625$ e 3,125 g i.a. ha $^{-1}$ ) equivalente a $0 ; 0,3906$; 0,$78125 ; 1,5625$ e $3,125 \%$ da dose recomendada pelo fabricante. Testaram-se onze espécies vegetais: arroz (Oryza sativa), aveia (Avena sativa), beterraba (Beta vulgaris), braquiária (Urochloa decumbens), feijão (Phaseolus vulgaris), girassol (Helianthus annuus), milho (Zea mays), pepino (Cucumis sativus), soja (Glycine max), tomate (Lycopersicon esculentum) e trigo (Triticum aestivum).
Após a semeadura, os vasos foram irrigados mantendo-se os teores de umidade próximo a capacidade de campo e depois de duas horas para procedeu-se à aplicação dos herbicidas. Para cada espécie foram colocadas cinco sementes por vaso à profundidade de 1-2 $\mathrm{cm}$ e após a emergência foi feito desbaste, deixando-se duas plantas por vaso. Durante a condução dos experimentos a irrigação dos vasos foi realizada sempre que necessária, visando manter a umidade necessária para o desenvolvimento das plantas.

A aplicação do herbicida foi realizada sobre o solo úmido utilizando-se um pulverizador instalado em ambiente controlado, provido de uma barra de pulverização com quatro pontas de pulverização XR $110.02 \mathrm{VS}$, espaçadas entre si em $0,5 \mathrm{~m}$ e posicionadas a $0,5 \mathrm{~m}$ de altura em relação à superfície das unidades experimentais. $\mathrm{O}$ sistema foi operado com velocidade de deslocamento de $3,6 \mathrm{~km} \mathrm{~h}^{-1}$, 
com volume de calda de $200 \mathrm{~L} \mathrm{ha}^{-1}$, pressão constante de 3,0

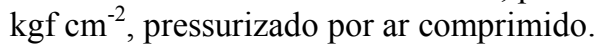

Aos 3, 7, 14 e 21 dias após a emergência (DAE) foi realizada a medição da altura de plantas em centímetros (ALT), com auxílio de régua milimetrada e a avaliação de fitointoxicação através de escala de notas na qual $0 \%$ representa nenhuma injúria e 100\% morte das plantas, conforme a metodologia proposta pela SBCPD (1995). Aos 21 DAE realizou-se a coleta da parte aérea e a raiz das plantas e secadas em estufa $\left(72 \mathrm{~h}\right.$, a $\left.65^{\circ} \mathrm{C}\right)$, visando à determinação da matéria seca de parte aérea (MSPA), de raiz (MSR) e total (MST).

Os dados obtidos foram submetidos à análise de variância $(p \leq 0,05)$ com auxílio do software Sisvar (FERREIRA, 2014) e ajustados ao modelo de regressão nãolinear logístico de três parâmetros por meio do pacote estatístico Sigmaplot versão 12.0, (Eq.1). Para o ajuste dos modelos os dados foram transformados em porcentagem em relação a testemunha, para as variáveis resposta fitointoxicação e altura, separou-se as espécies avaliadas e foi ajustado um modelo para cada período de avaliação, sendo considerado como fator a dose do herbicida, por outro lado, quando a variável resposta foi a matéria seca de parte aérea (MSPA), de raiz (MSR) e total (MST) o ajuste do modelo foi realizado para cada espécie em separado.

$$
y=\frac{a}{\left[1+\left(\frac{x}{b}\right)^{c}\right]}
$$

Onde: $\mathrm{y}=$ percentagem de altura, fitointoxicação, gramas de matéria seca da parte aérea, raiz e total; $x=$ dose do herbicida (g i.a. ha ${ }^{-1}$ ); a, b e c são parâmetros da equação, sendo que $\mathrm{a}=$ assíntota entre o ponto máximo e mínimo da variável, $\mathrm{b}=$ é a dose que proporciona $50 \%$ de resposta da variável.

\section{Resultados e discussão}

Foram observados diferentes níveis de tolerância das espécies após a aplicação do herbicida indaziflam nos dois experimentos. Ademais, os valores das variáveis analisadas fitointoxicação, altura (ALT), massa seca da parte aérea (MSPA), raiz (MSR) e total (MST) foram inversamente proporcionais ao aumento da dose do herbicida indaziflam.

As plantas que apresentaram elevada sensibilidade em relação ao herbicida foram: beterraba (Beta vulgaris), braquiária (Urochloa decumbens), girassol (Helianthus annuus), pepino (Cucumis sativus) e tomate (Lycopersicon esculentum). Para espécies se observou que mesmo na menor dose $(6,25$ g i.a. ou $6,25 \%$ da dose recomendada do herbicida indaziflam) não houve a emergência das plântulas, sendo realizado um segundo experimento.

$\mathrm{Na}$ avaliação de fitointoxicação tanto no primeiro quanto no segundo experimento para todas as espécies estudadas os sintomas iniciaram aos 3 DAA mesmo na menor dose do indaziflam $\left(6,25\right.$ e 0,3906 g i.a. ha ${ }^{-1}$, experimento 1 e 2, respectivamente), e se intensificaram no decorrer dos períodos de avaliação (Figuras 1 e 2).

Nas plantas monocotiledôneas verificou-se sintomas de fitointoxicação, caracterizados pelo enrolamento seguido de clorose e necrose das folhas (Figura 3), além de reduções mais acentuadas na ALT, MSPA, MSR e MST em braquiária (Urochloa decumbens), arroz (Oryza sativa), milho (Zea mays), trigo (Triticum aestivum) e aveia (Avena sativa), respectivamente. Importante ressaltar que no primeiro experimento, não houve a emergência das plântulas de $U$. decumbens na menor dose $(6,25 \%$ da dose recomendada e no segundo, aos 3 DAE com 1,5625 g i.a. ha ${ }^{-1}$ ) já foi observada morte das plantas (Figura 2), demonstrando maior sensibilidade dessa espécie.

Em plantas de arroz aos 14 DAE houve morte na maior dose $\left(100 \mathrm{~g}\right.$ i.a. ha $\left.{ }^{-1}\right)$ e aos 21 DAE na menor dose $\left(6,25 \mathrm{~g}\right.$ i.a. $\left.\mathrm{ha}^{-1}\right)$ os efeitos fitotóxicos chegaram a $63 \%$ (Figura 1). Os sintomas de fitointoxicação nas plantas de milho, trigo e aveia, foram considerados severos, chegando a $95 \%, 92 \%$ e $90 \%$, respectivamente, aos 21 DAE na dose recomendada pelo fabricante. 
R. C. DIAS et al.

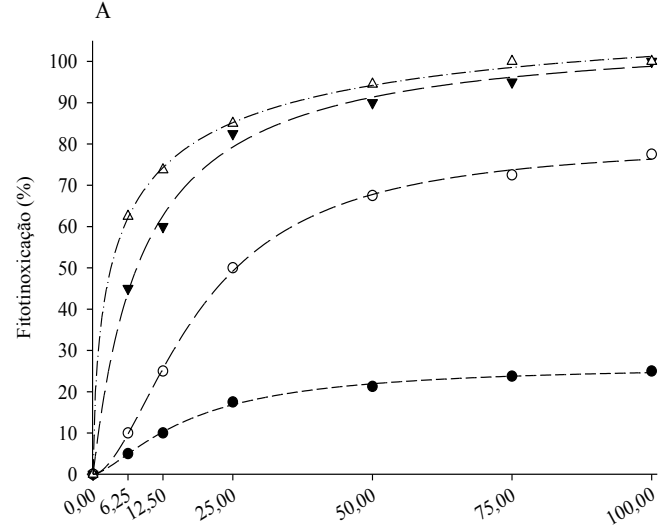

Doses de indaziflam (g i.a. ha $\left.{ }^{-1}\right)$

3 DAE $Y=26,51 / 1+(x / 19,34)^{\wedge}-1,46 R^{2}=0,99$

-Q- 7 DAE $Y=80,62 / 1+(x / 17,05)^{\wedge}-1,75 R^{2}=0,99$

$\checkmark-14 \mathrm{DAE} Y=107,58 / 1+(\mathrm{x} / 8,98)^{\wedge}-1,00 \mathrm{R}^{2}=0,99$

$-\triangle 21$ DAE $Y=117,79 / 1+(\mathrm{x} / 5,22)^{\wedge}-0,61 \mathrm{R}^{2}=0,99$

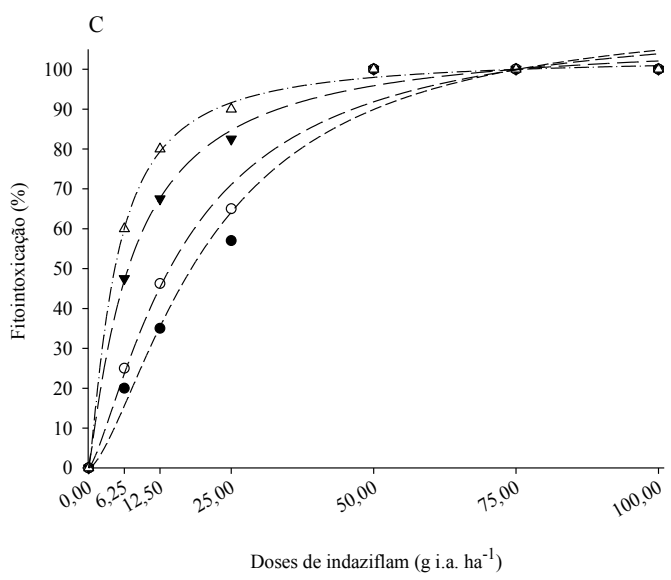

- 3 DAE $Y=115,28 / 1+(\mathrm{x} / 21,50)^{\wedge}-1,50 \mathrm{R}^{2}=0,98$

- - 7 DAE $Y=113,72 / 1+(x / 17,03)^{\wedge}-1,33 R^{2}=0,98$

$\rightarrow 14 \mathrm{DAE} \mathrm{Y}=108,09 / 1+(\mathrm{x} / 7,89)^{\wedge}-1,11 \mathrm{R}^{2}=0,99$

$-\triangle-21$ DAE $Y=103,13 / 1+(x / 4,78)^{\wedge}-1,25 R^{2}=0,99$

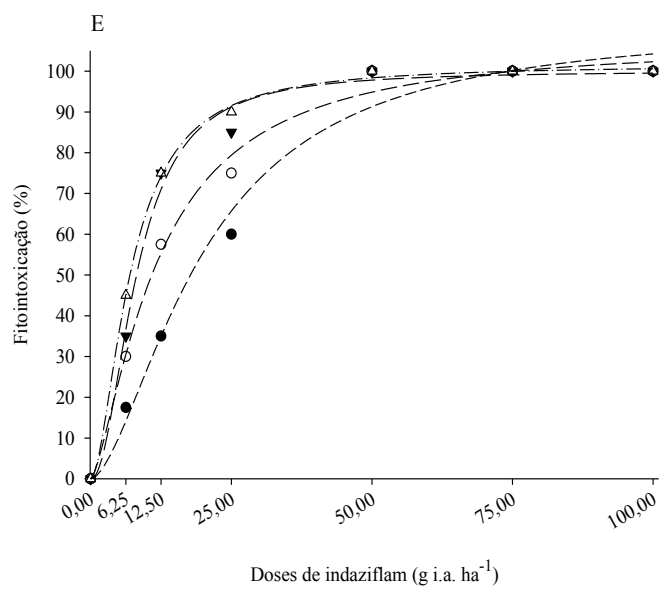

-. 3 DAE $Y=111,69 / 1+(x / 20,13)^{\wedge}-1,64 R^{2}=0,98$

-Q. 7 DAE $Y=107,37 / 1+(x / 11,97)^{\wedge}-1,41 R^{2}=0,99$

$\longrightarrow-14$ DAE $Y=100,07 / 1+(\mathrm{x} / 8,13)^{\wedge}-2,08 \mathrm{R}^{2}=0,99$ $\checkmark 21$ DAE $Y=101,58 / 1+(\mathrm{x} / 7,08)^{\wedge}-1,75 \mathrm{R}^{2}=0,99$

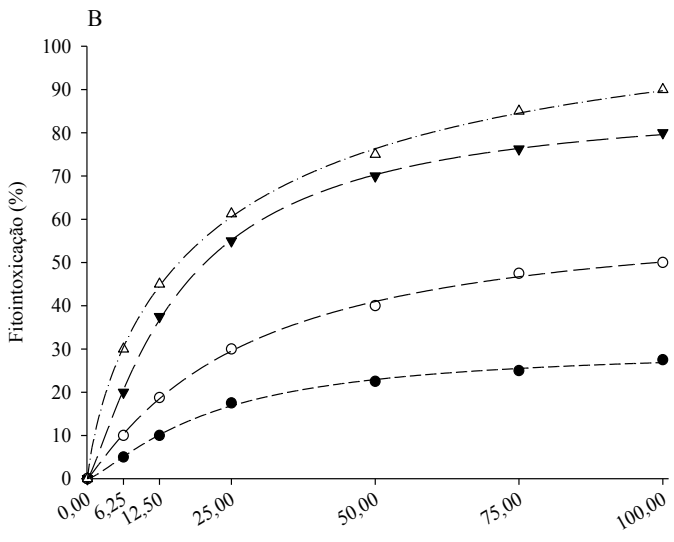

Doses de indaziflam (g i.a. ha ${ }^{-1}$ )

-. 3 DAE $Y=30,38 / 1+(x / 27,66)^{\wedge}-1,31 R^{2}=0,99$ - $\cdot 7$ DAE $Y=62,53 / 1+(x / 23,17)^{\wedge}-1,08 R^{2}=0,99$ - 14 DAE $\mathrm{Y}=88,31 / 1+(\mathrm{x} / 21,10)^{\wedge}-1,22 \mathrm{R}^{2}=0,99$ - 21 DAE $Y=117,60 / 1+(\mathrm{x} / 16,48)^{\wedge}-0,80 \mathrm{R}^{2}=0,99$
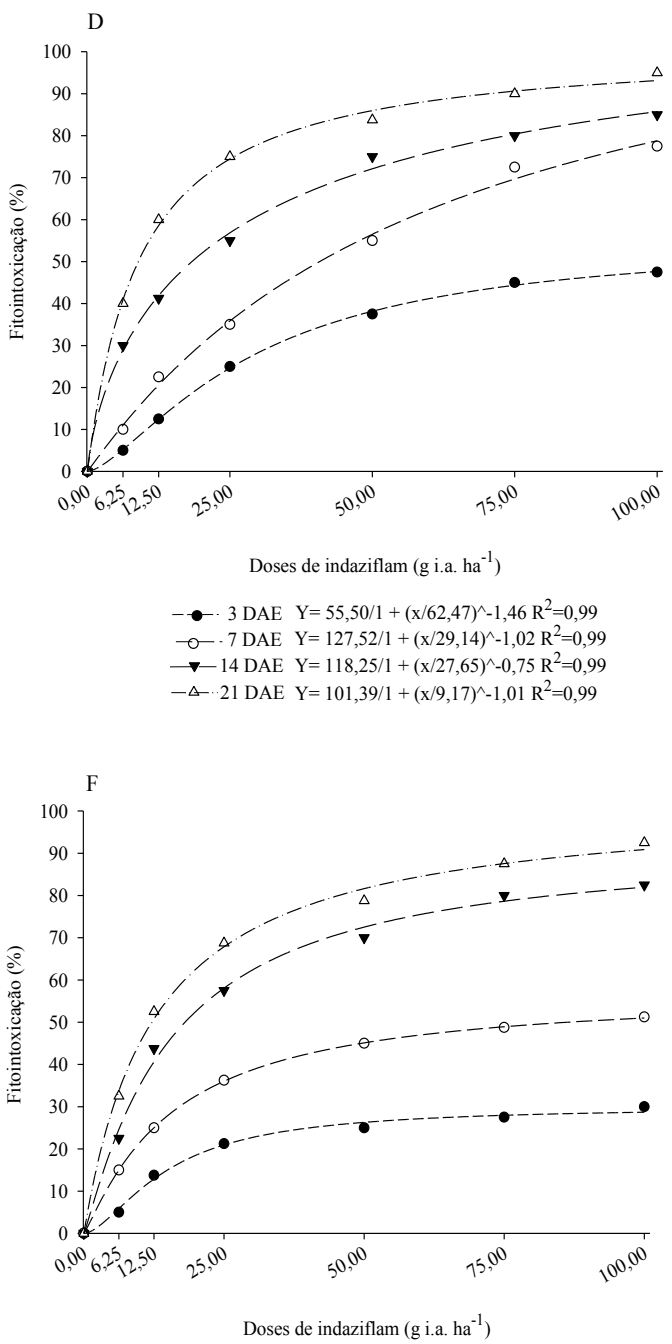

- $3 \mathrm{DAE} \quad \mathrm{Y}=30,01 / 1+(\mathrm{x} / 15,66)^{\wedge}-1,62 \mathrm{R}^{2}=0,98$ $7 \mathrm{DAE} \quad \mathrm{Y}=57,33 / 1+(\mathrm{x} / 15,53)^{\wedge}-1,12 \mathrm{R}^{2}=0,98$ - 14 DAE $Y=92,36 / 1+(x / 14,87)^{\wedge}-1,11 R^{2}=0,99$ - $\cdots 21$ DAE $\quad \mathrm{Y}=102,57 / 1+(\mathrm{x} / 12,73)^{\wedge}-0,99 \mathrm{R}^{2}=0,99$

Figura 1. Fitointoxicação (\%) de plantas de arroz - A, aveia - B, feijão - C, milho - D, soja - E e trigo - F submetidas a aplicação de 0; 6,25; 12,$5 ; 25 ; 50 ; 75 ; 100 \%$ da dose recomendada de indaziflam. Primeiro experimento 
R. C. DIAS et al.
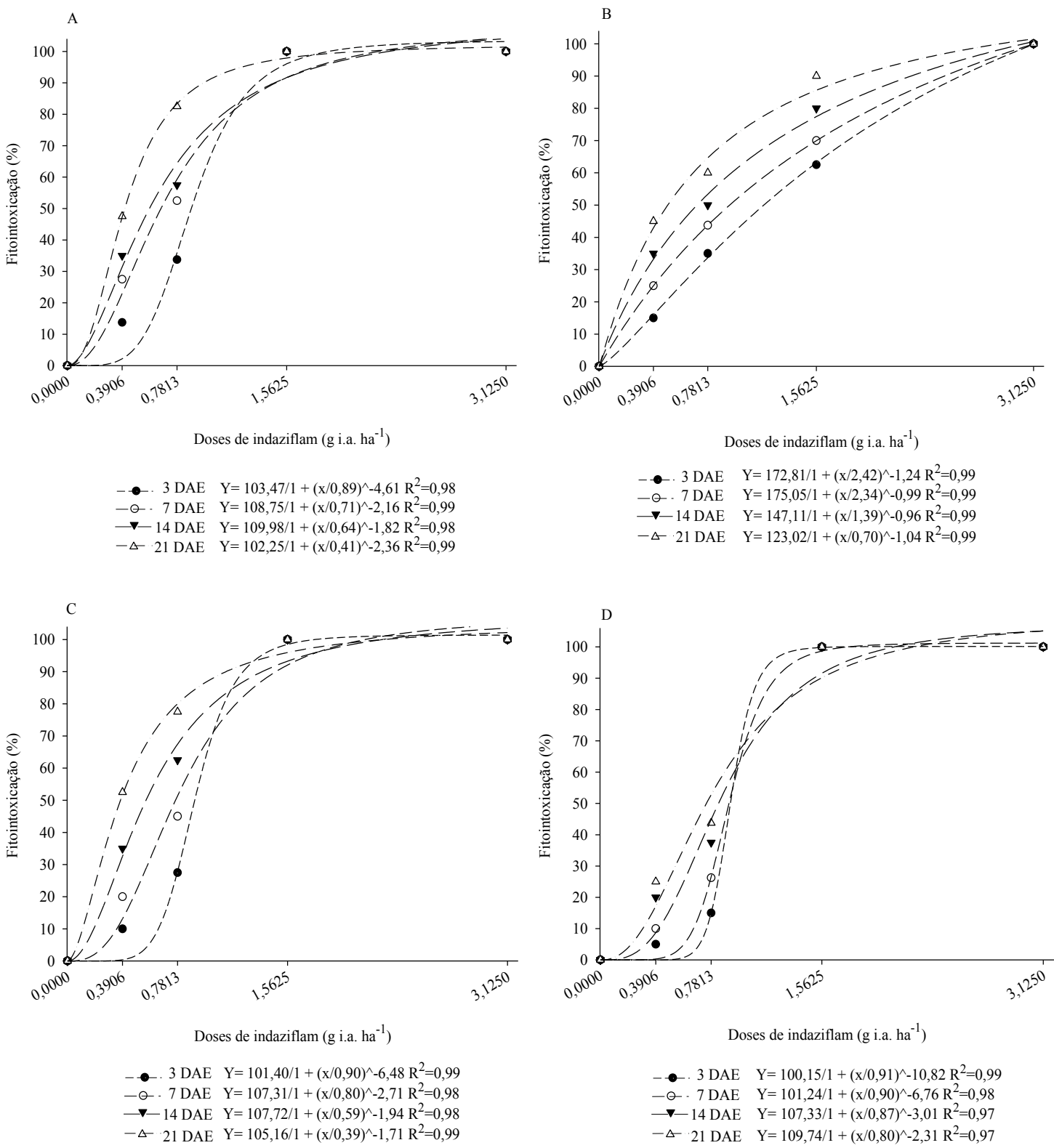

Figura 2. Fitointoxicação (\%) de plantas de braquiária - A, girassol - B, pepino - C e tomate - D submetidas a aplicação de 0; 0,3906; 0,$7813 ; 1,5625$ e $3,1250 \%$ da dose recomendada de indaziflam. Segundo experimento.

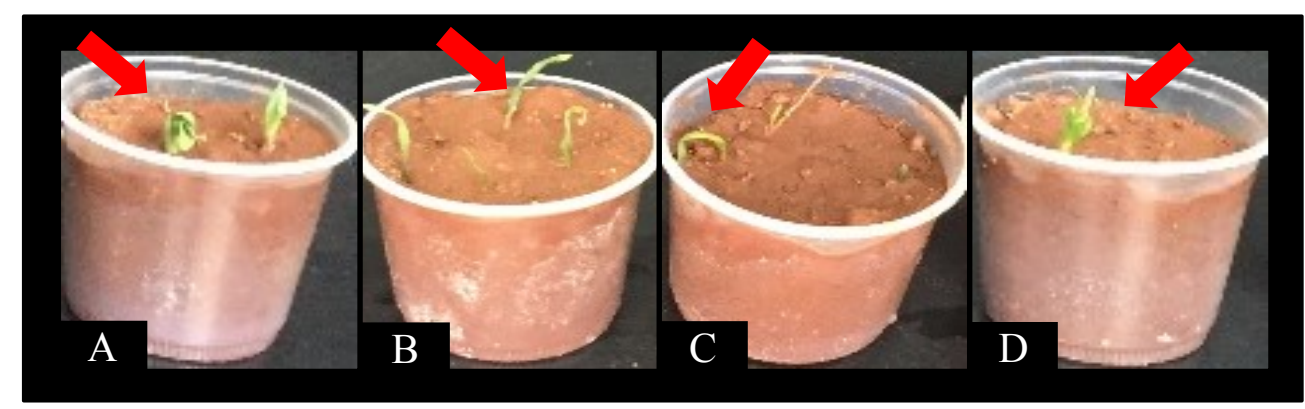

Figura 3. Sintomas de fitointoxicação em espécies monocotiledôneas (Milho A e D; Trigo B e Aveia C) causado pela aplicação do herbicida indaziflam (50,00 g i.a. ha $\left.{ }^{-1}\right)$. Foto: Roque de Carvalho Dias. 
Analisando as espécies eudicotiledôneas, a beterraba, girassol, pepino e tomate não emergiriam no primeiro estudo em que a menor dose foi de $6,25 \mathrm{~g} \mathrm{ha}^{-1}$ de indaziflam. Já em relação ao feijão e soja, notou-se valores similares para as variáveis estudadas, fitointoxicação, ALT, MSPA, MSR e MST (Figuras 1, 5, e 7). Vale destacar também que as doses superiores a $50 \mathrm{~g}$ i.a. ha $^{-1}$ do indaziflam foram suficientes para provocar a morte dessas espécies. De maneira geral, os efeitos nessas plantas foram caracterizados pelo espessamento e rompimento do hipocótilo (Figura 4). Em plantas de beterraba, no segundo estudo, mesmo na dose de 0,3906 i.a. ha ${ }^{-1}$ não foi observada a emergência dessa espécie, este comportamento classifica como espécie extremamente sensível ao herbicida. Em pepino e tomate foram observadas morte das plantas na dose de 1,5625 i.a. ha $^{-1}$ e em girassol 3,125 i.a. ha ${ }^{-1}$ aos 3 DAE (Figura 2).

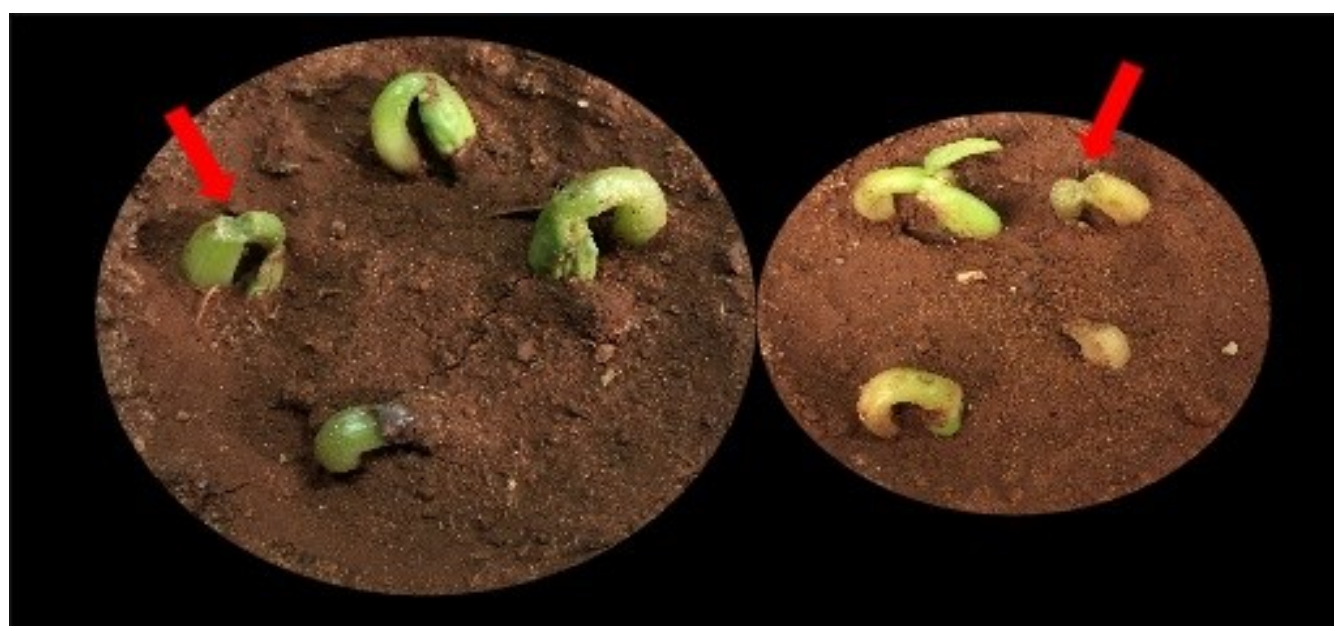

Figura 4. Sintomas de fitointoxicação em plantas de feijão submetidos pela aplicação do herbicida indaziflam $\left(6,25 \mathrm{~g}_{\text {i.a. ha }}{ }^{-1}\right)$. Foto: Roque de Carvalho Dias.

Os sintomas verificados no presente estudo devem-se ao mecanismo de ação desse herbicida, Brabham et al. (2014) salientaram que o indaziflam atua na inibição da biossíntese de celulose. De acordo com esses autores, isso ocorre pelo fato dessa molécula impedir a polimerização de celulose a partir do substrato UDP-glicose, realizada pela glucosiltransferase e, também, pela inibição da multiplicação das células, a partir de outros polissacarídeos, devido ao acúmulo de ácido nítrico nas mesmas. Ainda nesse trabalho, observaram que a presença do indaziflam inibiu a incorporação do $\mathrm{C} 14$ da glicose em ácidos insolúveis da fração de celulose, o qual contribui para formação da parede celular. Além disso, a inibição da divisão celular do tecido meristemático, também, tem sido proposta como modo de ação secundário (GRIFFIN, 2005). De acordo com Kaapro e Hall (2012), a inibição da deposição de cristais na parede celular afeta a formação, a divisão e o alongamento celular, entretanto, tecidos completamente desenvolvidos não são afetados. Logo, os sintomas em plantas sensíveis são caracterizados pela ausência de emergência.

Guerra et al. (2013) relataram que a beterraba, o girassol e o pepino têm elevada sensibilidade ao indaziflam. Em solo aplicado com indaziflam (20 g i.a. ha $\left.{ }^{-1}\right)$, constatouse que as plântulas de beterraba e pepino não emergiram
Guerra et al. (2014), demonstrando serem espécies importantes na detecção desse herbicida no solo. Já Jeffries et al. (2014), simulando deriva após 70 dias depois a aplicação de $1,8 \mathrm{~g} \mathrm{ha}^{-1}$ de indaziflam observaram efeitos negativos em plantas de tomate.

Além de sensível ao indaziflam, a beterraba apresenta -se como sensível a herbicidas de variados mecanismos de ação como sulfentrazone (BLANCO et al., 2010), saflufenacil (DIESEL et al., 2012), e ethoxysulfuron (OLIVEIRA et al., 2018).

De maneira geral, a dose de $6,25 \mathrm{~g}$ i.a. ha $^{-1}$ foi suficiente em reduzir $73 \%$ da ALT aos 21 dias após a emergência (DAE) em arroz, milho e trigo em relação a testemunha. (Figura 5) e em capim-braquiária a redução foi de $45 \%$ já aos 3 DAE na menor dose utilizada (Figura 6). A presença de indaziflam no solo ocasionou elevada redução em ALT em feijão e soja, com valores próximos a $45 \%$ aos 21 DAE na menor dose estudada $\left(6,25 \mathrm{~g}\right.$ i.a. ha $\left.^{-1}\right)$ (Figura 5), $50 \%$ em pepino e tomate $\left(0,78125 \mathrm{~g}\right.$ i.a. ha $\left.^{-1}\right)$ e $46 \%$ em girassol $\left(1,5625 \mathrm{~g}\right.$ i.a. ha $\left.{ }^{-1}\right)$ (Figura 6). Jeffries et al. (2014), verificaram reduções na altura das plantas sob simulação de deriva do herbicida indaziflam e que essas reduções foram acompanhadas dos sintomas de fitointoxicação, corroborando os resultados desse trabalho. 
R. C. DIAS et al.

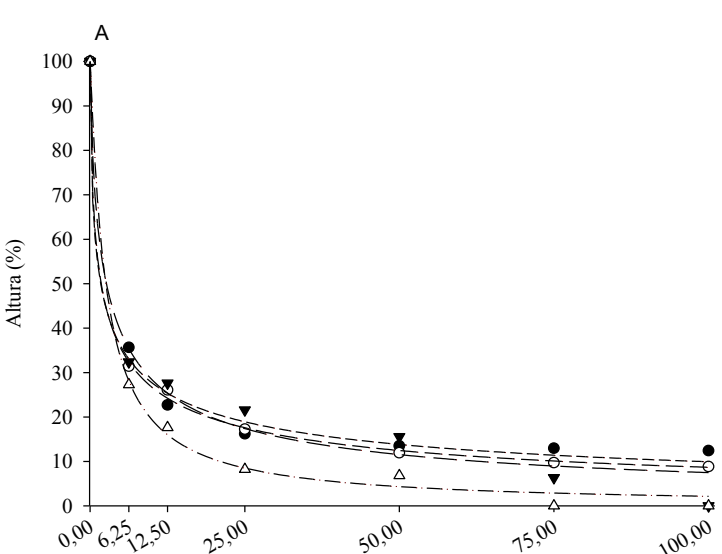

Doses de indaziflam (g i.a. ha $^{-1}$ )

- 3 DAE $\mathrm{Y}=100,00 / 1+(\mathrm{x} / 2,62)^{\wedge} 0,54 \mathrm{R}^{2}=0,99$ - -7 DAE $\quad \mathrm{Y}=100,00 / 1+(\mathrm{x} / 2,52)^{\wedge} 0,58 \mathrm{R}^{2}=0,99$

$\checkmark-14 \mathrm{DAE} \quad \mathrm{Y}=100,00 / 1+(\mathrm{x} / 1,77)^{\wedge} 0,69 \mathrm{R}^{2}=0,99$

$-\triangle 21 \mathrm{DAE} \quad \mathrm{Y}=100,00 / 1+(\mathrm{x} / 1,69)^{\wedge} 0,99 \mathrm{R}^{2}=0,99$

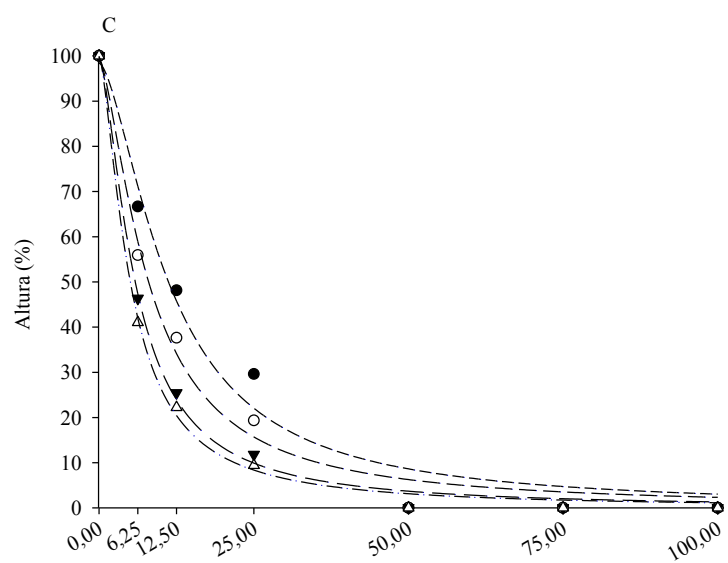

Doses de indaziflam (g i.a. $\mathrm{ha}^{-1}$ )

- 3 DAE $\mathrm{Y}=100,00 / 1+(\mathrm{x} / 11,44)^{\wedge} 1,58 \mathrm{R}^{2}=0,98$

- $\mathrm{Q}-7 \mathrm{DAE} \quad \mathrm{Y}=100,00 / 1+(\mathrm{x} / 8,06)^{\wedge} 1,47 \mathrm{R}^{2}=0,99$

$\checkmark-14 \mathrm{DAE} \quad \mathrm{Y}=100,00 / 1+(\mathrm{x} / 5,84)^{\wedge} 1,51 \mathrm{R}^{2}=0,99$

$-\triangle 21 \mathrm{DAE} \quad \mathrm{Y}=100,00 / 1+(\mathrm{x} / 5,03)^{\wedge} 1,49 \mathrm{R}^{2}=0,99$

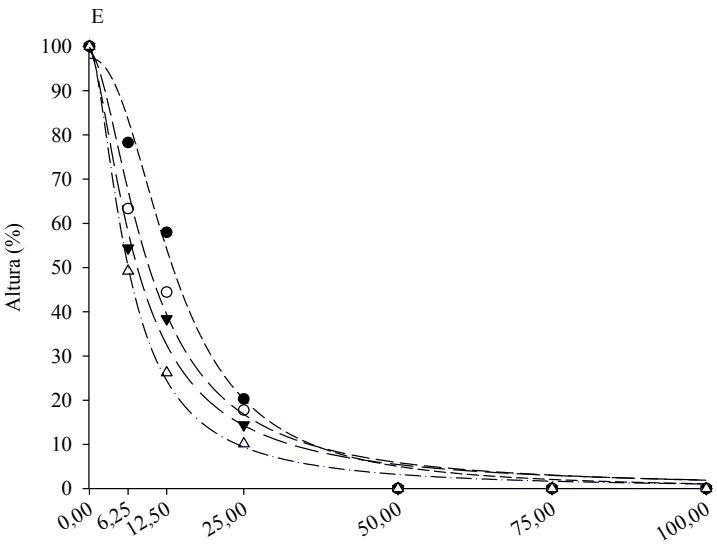

Doses de indaziflam (g i.a. ha ${ }^{-1}$ )

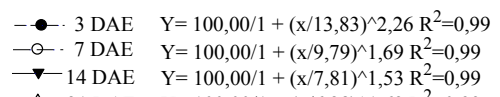

$-\triangle \cdot 21 \mathrm{DAE}$

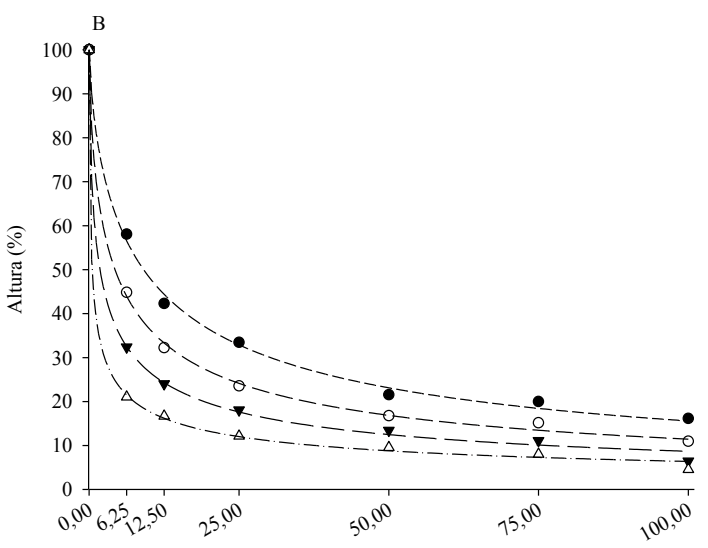

Doses de indaziflam $\left(\mathrm{g}\right.$ i.a. ha $\left.{ }^{-1}\right)$

- $3 \mathrm{DAE} \quad \mathrm{Y}=100,00 / 1+(\mathrm{x} / 9,05)^{\wedge} 0,81 \mathrm{R}^{2}=0,99$

-Q- $7 \mathrm{DAE} \quad \mathrm{Y}=100,00 / 1+(\mathrm{x} / 4,29)^{\wedge} 0,72 \mathrm{R}^{2}=0,99$

$\rightarrow-14 \mathrm{DAE} \quad \mathrm{Y}=100,00 / 1+(\mathrm{x} / 1,79)^{\wedge} 0,70 \mathrm{R}^{2}=0,99$

$-\triangle 21$ DAE $Y=100,00 / 1+(x / 0,46)^{\wedge} 0,64 R^{2}=0,99$

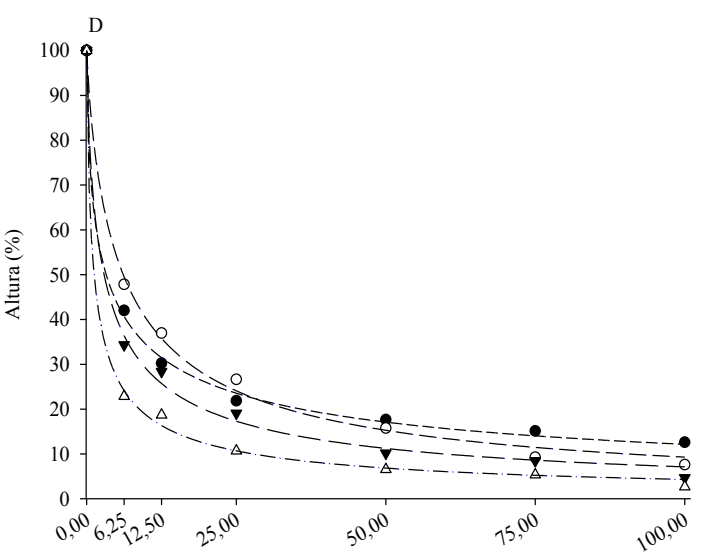

Doses de indaziflam (g i.a. ha $\left.{ }^{-1}\right)$

•- 3 DAE $\mathrm{Y}=100,00 / 1+(\mathrm{x} / 6,12)^{\wedge} 0,57 \mathrm{R}^{2}=0,99$ Q- $7 \mathrm{DAE} \quad \mathrm{Y}=100,00 / 1+(\mathrm{x} / 3,24)^{\wedge} 0,81 \mathrm{R}^{2}=0,99$ $\longrightarrow 14 \mathrm{DAE} Y=100,00 / 1+(\mathrm{x} / 2,90)^{\wedge} 0,72 \mathrm{R}^{2}=0,99$ $-\triangle 21 \mathrm{DAE} Y=100,00 / 1+(\mathrm{x} / 1,22)^{\wedge} 0,70 \mathrm{R}^{2}=0,99$

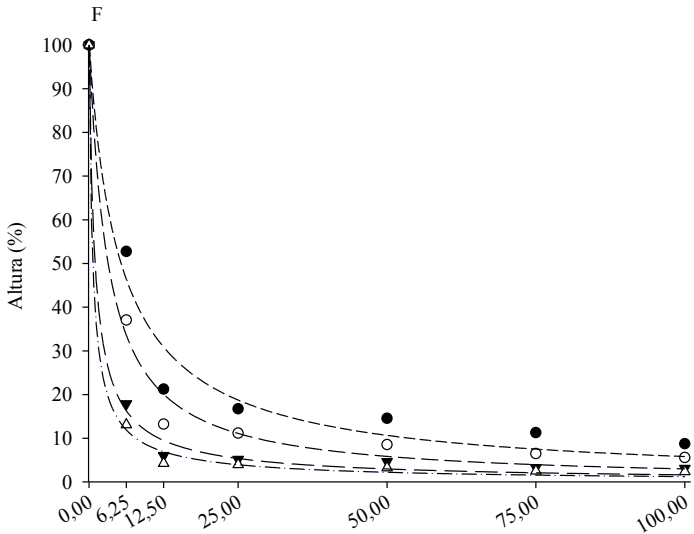

Doses de indaziflam (g i.a. ha ${ }^{-1}$ )

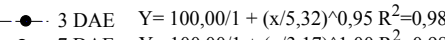

-Q. 7 DAE $\quad \mathrm{Y}=100,00 / 1+(\mathrm{x} / 3,17)^{\wedge} 1,00 \mathrm{R}^{2}=0,98$

$\rightarrow-14 \mathrm{DAE} \quad \mathrm{Y}=100,00 / 1+(\mathrm{x} / 0,98)^{\wedge} 0,88 \mathrm{R}^{2}=0,99$

$-\triangle-21$ DAE $\quad \mathrm{Y}=100,00 / 1+(\mathrm{x} / 0,60)^{\wedge} 0,85 \mathrm{R}^{2}=0,99$

Figura 5. Altura (\%) em relação ao tratamento controle (sem herbicida) de plantas de arroz - A, aveia - B, feijão - C, milho - D, soja - E e trigo - F submetidas a aplicação de $0 ; 6,25 ; 12,5 ; 25 ; 50 ; 75 ; 100 \%$ da dose recomendada de indaziflam. Primeiro experimento. 

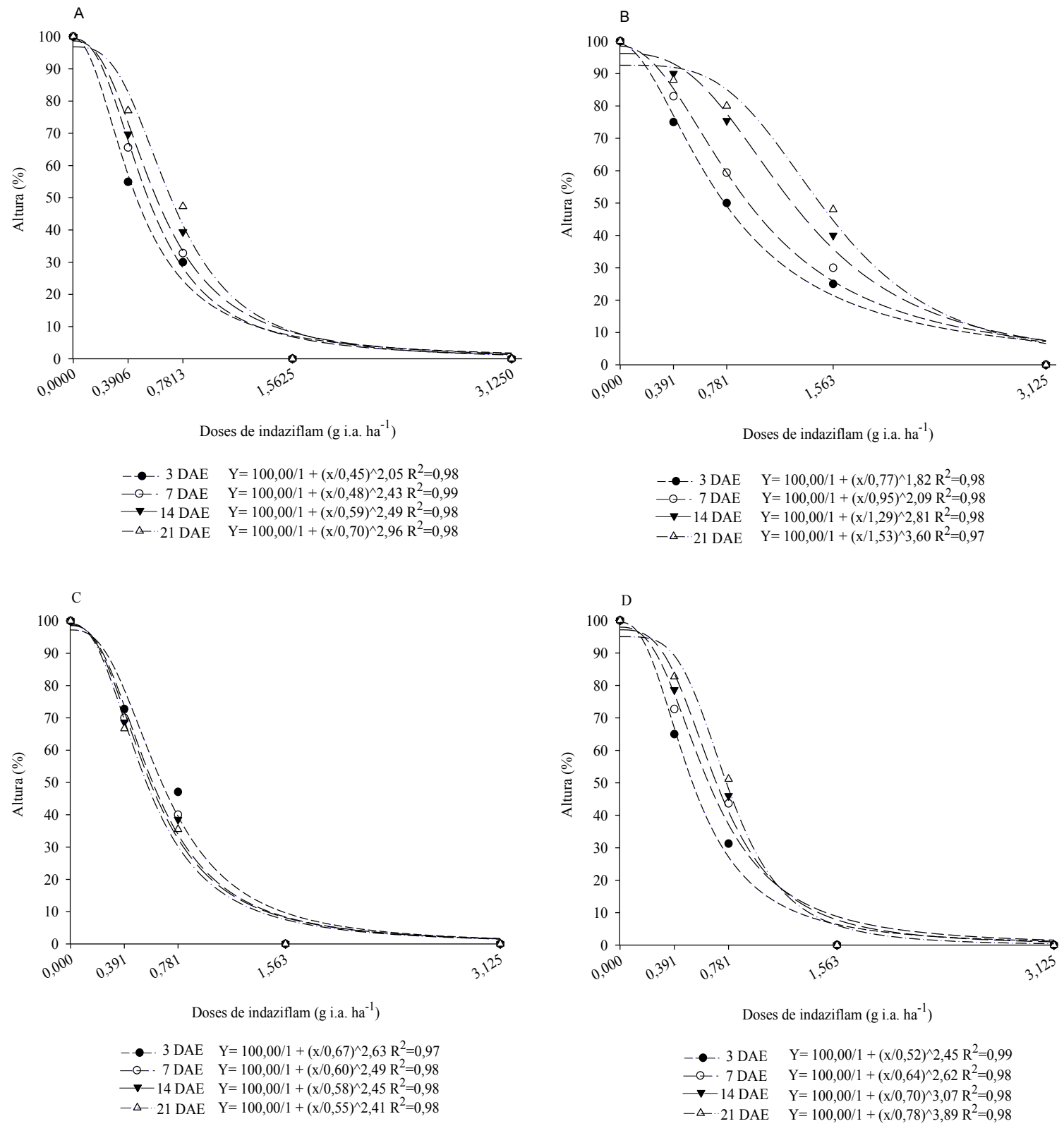

Figura 6. Altura (\%) em relação ao tratamento controle (sem herbicida) de plantas de braquiária - A, girassol - B, pepino - C e tomate - D submetidas a aplicação de $0 ; 0,3906 ; 0,7813 ; 1,5625$ e 3,1250\% da dose recomendada de indaziflam. Segundo experimento.

Quanto à MSPA, MSR e total MST (Figuras 7 e 8), observa-se que plantas de capim-braquiária apresentaram valores de $I_{50} 0,86 ; 0,50$ e 0,60 g i.a. ha ${ }^{-1}$, respectivamente, entretanto, vale salientar que as demais espécies monocotiledôneas utilizadas no presente estudado também apresentaram também valores do $I_{50}$ baixos. Dessa maneira, $U$. decumbens apresentou maior sensibilidade em espécies monocotiledôneas seguido por $O$. sativa, Z. mays, $T$. aestivum e $A$. sativa. Já os valores de $I_{50}$ em relação a MSPA, MSR e MST foram inferiores a 9,05 g i.a. ha ${ }^{-1}$ do indaziflam em feijão e soja (Figura 7). Nessas mesmas variáveis, no segundo experimento, observou maiores reduções em tomate, pepino e girassol, apresentando valores de $I_{50}$ de MST $0,56,0,73$ e 0,88 g i.a. ha $^{-1}$ respectivamente (Figura 8). Assim, em eudicotiledôneas a L. esculentum demonstrou maior sensibilidade seguido de $C$. sativus e $H$. annuus. Tendo em vista, que em B. vulgaris houve morte da planta nos dois experimentos, este comportamento classifica a beterraba como espécie extremamente sensível ao herbicida, entretanto, dificulta sua seleção como planta bioindicadora por impossibilitar a visualização dos sintomas típicos do produto. 
R. C. DIAS et al.
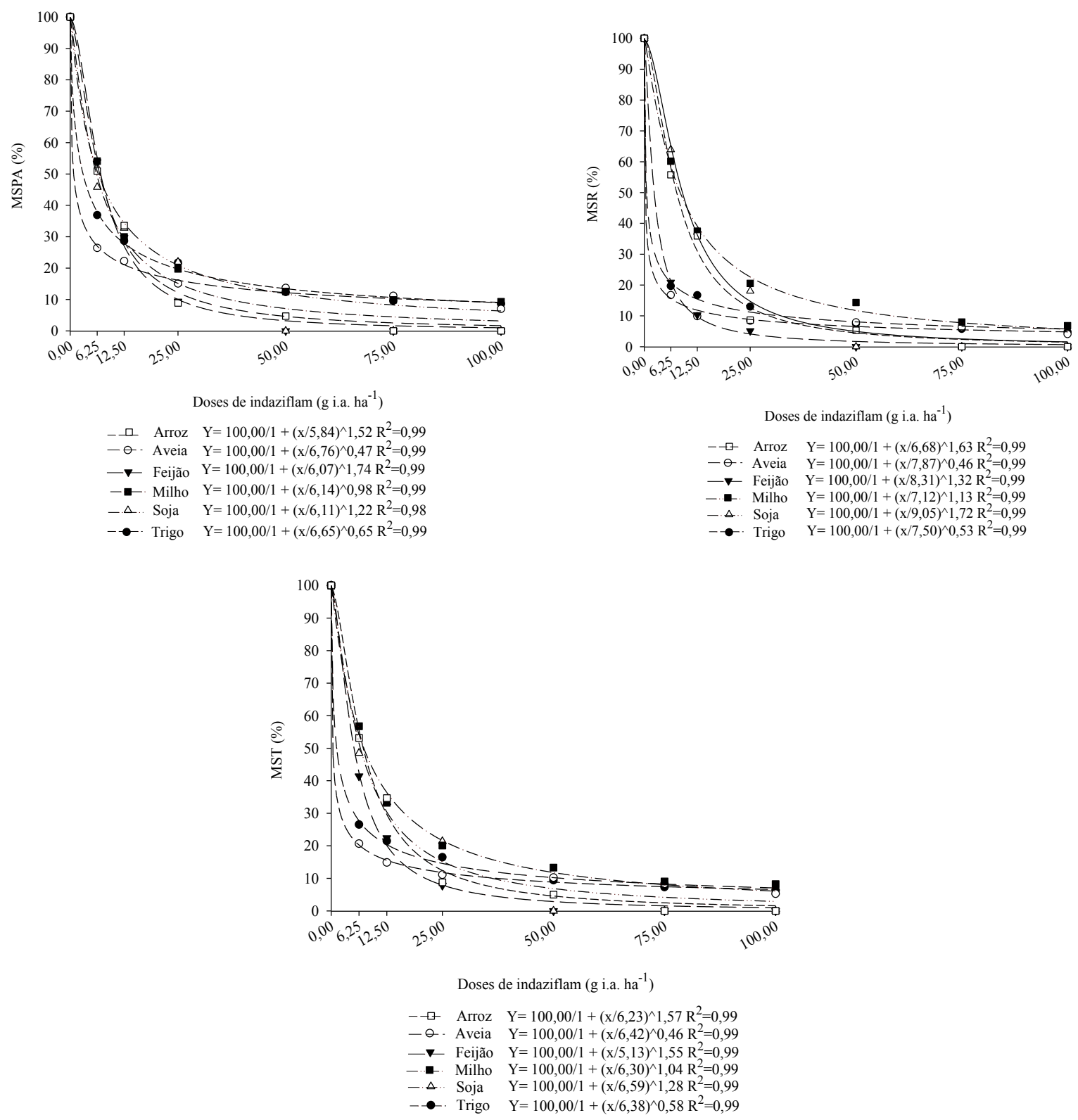

Figura 7. MSPA, MSR e MST (\%) de plantas de arroz, aveia, feijão, milho, soja e trigo submetidas a aplicação de $0 ; 6,25 ; 12,5 ; 25 ; 50 ; 75$; $100 \%$ da dose recomendada de indaziflam. Primeiro experimento. 

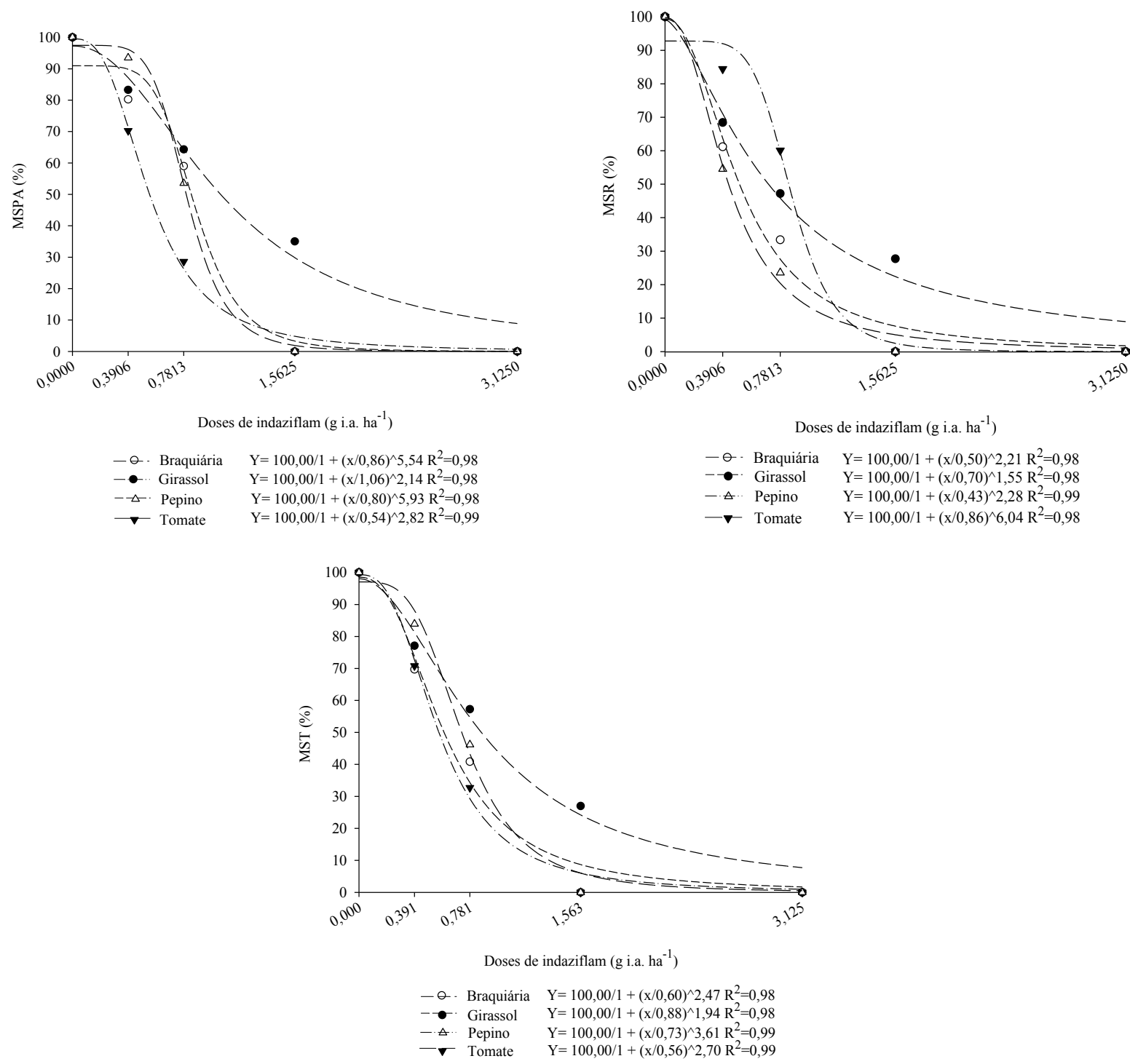

Figura 8. MSPA, MSR e MST (\%) de plantas de braquiária, girassol, pepino e tomate submetidas a aplicação de $0 ; 0,3906 ; 0,7813 ; 1,5625$ e $3,1250 \%$ da dose recomendada de indaziflam. Segundo experimento.

As reduções na MSPA, MSR e MST apresentaram relação direta aos níveis de fitointoxicação proporcionados pelo herbicida às plantas estudadas. $\mathrm{O}$ indaziflam é absorvido pelas raízes e pelos tecidos da parte aérea, inibindo o crescimento da raiz por meio da interferência na formação da parede celular e na divisão celular, logo, com os danos no sistema radicular das espécies avaliadas houve consequentemente redução no crescimento da parte aérea e total das plantas. Segundo Jeffries et al. (2014), mostram que a redução de matéria seca de parte aérea, raiz, altura de plantas e injúrias das plantas são a taxas semelhantes, comprovando comportamento análogo entre os órgãos das plantas testadas neste estudo.

\section{Conclusão}

Entre as espécies avaliadas em ordem de acordo com o potencial uso como bioindicadora do herbicida indaziflam aplicado no solo, em monocotiledôneas: Urochloa decumbens, Oryza sativa, Zea mays, Triticum aestivum e Avena sativa, em eudicotiledôneas: Lycopersicon esculentum, Cucumis sativus, Helianthus annuus, Phaseolus vulgaris e Glycine max. Embora a espécie B. vulgaris ser extremamente sensível ao herbicida, sua seleção como planta bioindicadora deve ser analisada para cada situação.

\section{Referências}

Alonso, D.G.; Oliveira Jr, R.S.; Hall, K.E.; Koskinen, W.C.; Constantin, J.; Mislankar, S. Changes in sorption of indaziflam and three transformation products in soil with aging. Geoderma, v.239, n.4, p.250-256, 2015.

Amim, R.T.; Freitas, S.P.; Freitas, I.L.J.; Gravina, G.A.; Paes, H.M.F. Controle de plantas daninhas pelo indaziflam em solos com diferentes características físico-químicas. Planta Daninha, v.32, n.4, p.791-800, 2014. 
Blanco, F.M.G.; Velini, E.D.; Filho, A.B. Persistência do herbicida sulfentrazone em solo cultivado com cana-deaçúcar. Bragantia, v.69, n.1, p.71-75, 2010.

Brabham, C.; Lei, L.; Gu, Y.; Stork, J.; Barrett, M.; Debolt, S. Indaziflam herbicidal action: a potent cellulose biosynthesis inhibitor. Plant Physiology, v.166, n.3, p.1177$1185,2014$.

Dias, R.C.; Mendes, K.F.; Oliveira, C.R.; Pucci, L.F.; Reis, M.R. Métodos de Análises e Comportamento de Herbicidas no Solo. In: Lopes, E.A et al. (Ed.). A Química na Produção Vegetal. 1 ed. Rio Paranaíba, MG: Editora dos Autores, 2017. p.339-399.

Diesel, F.; Trezzi, M.M.; Pazuch, D.; Xavier, E.; Rosin, D.; Pagnoncelli, F. Seleção de espécies da família Cucurbitaceae e Chenopodiaceae para indicação da presença de saflufenacil no solo. Pesticidas: Revista de Ecotoxicologia e Meio Ambiente, v.22, p.35-42, 2012.

Empresa Brasileira de Pesquisa Agropecuária - EMBRAPA. Centro Nacional de Pesquisa Agropecuária de Solos (Brasília, DF). Sistema brasileiro de classificação de solos. Brasília: Embrapa Produção de Informação; Brasília, DF: Embrapa Solos, 2013. 154p.

Ferreira, D.F. Sisvar: a guide for its bootstrap procedures in multiple comparisons. Ciência e Agrotecnolgia, v.38, n.2, p.109-112, 2014

Griffin, J.L. Inhibition of cell wall synthesis. In: Griffin, J. L. Weed course. 2005, p. 150-153.

Guerra, N.; de Oliveira Júnior, R.S.; Constantin, J.; de Oliveira Neto, A.M.; Braz, G.B.P. Aminocyclopyrachlor e indaziflam: Seletividade, controle e comportamento no ambiente. Revista Brasileira de Herbicidas, v.12, n.3, p.285-295, 2013.

Guerra, N.; Oliveira Neto, A.M.; Oliveira, J.R.; Constantin, J.; Takano, H.K. Sensibility of plant species to herbicides aminocyclopyrachlor and indaziflam. Planta Daninha, v,32, n.3, p.609-617, 2014.

Guerra, N.; Oliveira Junior, R.S.; Constantin, J.; Oliveira Neto, A.M.; Gemelli, A.; Pereira Junior, D. M.; Guerra, A. (2016). Persistence of Biological Activity and Leaching Potential of Herbicides Aminocyclopyrachlor and Indaziflam in Soils with Different Textures1. Planta Daninha, v.34, n.2, p.345-356, 2016.

Inoue, M.H.; Santana, C.T.C.; Oliveira, J.R.; Possamai, A.C.S.; Santana, D.C.; Arruda, R.A D.; Dallacort, R.; Sztoltz, C. L. Efeito residual de herbicidas aplicados em préemergência em diferentes solos. Planta Daninha, v.29, n.2, p.429-435, 2011.

Jeffries, M.D.; Mahoney, D.J.; Gannon, T.W. Effect of Simulated Indaziflam Drift Rates on Various Plant Species.
Weed Technology, v.28, n.4, p.608-616, 2014.

Kaapro, J.; Hall, J. Indaziflam, a new herbicide for preemergent control of weeds in turf, forestry, industrial vegetation and ornamentals. Pakistan Journal Weed Science Research, v.18, n.esp., p.267-270, 2012.

MAPA - Ministério da Agricultura. AGROFIT - Sistema de Agrotóxicos Fitossanitários. Disponível em: <http:// www.agricultura.gov.br/servicos-e-sistemas/sistemas/ agrofit $>>$ Acesso em: 03 jul. 2018.

Melo, C.A.D., Dias, R.C; Mendes, K.F.; Assis, A.C.D.L.P.; dos Reis, M.R. Carryover de herbicidas em sistemas cultivados com olerícolas. Revista Brasileira de Herbicidas, v.15, n.1, p.67-78, 2016.

Nunes, A.L.; Vidal, R.A. Seleção de plantas quantificadoras de herbicidas residuais. Pesticidas: Revista de Ecotoxicologia e Meio Ambiente, v.19, n.1, p.19-28, 2009.

Oliveira, T.L.; de Paula Senoski, M.; Assis, A.C.D.L.P.; de Miranda, V.P.; Melo, C.A.D.; dos Reis, M.R. Seleção de espécies bioindicadoras do herbicida ethoxysulfuron. Revista de Ciências Agrárias, v.61, n.2, 2018.

Sociedade Brasileira da Ciência das Plantas Daninhas SBCPD. Procedimentos para instalação, avaliação e análise de experimentos com herbicidas. Londrina: SBCPD, 1995. $42 \mathrm{p}$.

Tompkins, J. Environmental Protection Agency Pesticide Fact Sheet: Indaziflam. Environmental Protection Agency, 2010 . 